\title{
ACTIVIDAD BANCARIA DE LAS CIUDADES EN LA ÉPOCA CLÁSICA (SIGLOS I-III d.C.)*
}

BANKING OF CITIES IN THE CLASSICAL PERIOD (I- III CENTURY A.D.)

Andrea Trisciuoglio

Professore Associato di Diritto romano

Università di Torino (I) andrea.trisciuoglio@unito.it 


\section{Premisa.}

Las fuentes jurídicas, literarias y epigráficas nos enseñan con claridad que los préstamos que se refieren a las colonias y municipios en la edad clásica son de dos tipos: 1) aquellos en los que la parte pública, que necesitaba financiación externa, tenía el papel de mutuario1; 2) aquellos otros en los que la parte

* $\quad$ El presente artículo se enmarca dentro del proyecto de investigación: "Public and Fiscal Contracts in the Roman Experience" (Progetti di ricerca di Ateneo 2012 - call 2 - Università di Torino) subvencionado por la Compagnia di San Paolo.

1 Cfr. en particular el cap. 80 de la lex Irnitana que lleva la rúbrica de pecunia publice mutua sumenda; sobre este capítulo véase A.TORRENT, Financiación externa de los «municipia». Lex Irnitana cap. 80, en RDR. 10 (2010); para la bibliografía anterior cfr. A. BRICCHI, Amministratori ed actores. La responsabilità nei confronti dei terzi per l'attività negoziale degli agenti municipali, en Gli Statuti Municipali (a cura di L. Capogrossi Colognesi - E. Gabba), Pavia 2006, p. 356 nt. 62. La obligación de restituir de la civitas surge en el momento en que se ingresa el dinero en la caja pública municipal: cfr. D.12.1.27 (Ulp. 10 ad ed.): «Civitas mutui datione obligari potest, si ad utilitatem eius pecuniae versae sunt; alioquin ipsi soli qui contraxerunt, non civitas, tenebuntur»; A. BRICCHI, op. cit., p. 354 ss.; A. TRISCIUOGLIO, Societas publicanorum e aspetti della responsabilità esterna. Atti del Seminario di studi su: "Societas. Strumento di organizzazione pubblica e privata", Sassari, 4-5 maggio 2012, en Diritto@Storia 11 (2013), § 3.1. Se basan- creo-en este texto unas reflexiones de Rolandino de' Passaggieri (notario y jurisconsulto de Bononia, siglo XIII) que se refieren al Instrumentum mutui contracti a communi civitatis; al respecto véase $\mathrm{M}$. 
pública, que tenía una cierta disponibilidad de capital y con ello la posibilidad de hacer inversiones, asumía el papel de prestamista2. Voy a detenerme brevemente en el procedimiento decisorio y los medios de control que facilitaban rentabilizar de la mejor manera posible el capital en dinero de los municipia, los

TUCCI, L'amministrazione tra pubblico e privato e il principio di legalità dall'antichità ai giorni nostri. Aspetti ricostruttivi e prospettive di sviluppo, Milano 2008, p. 31 s.

2 Las fuentes relevantes son mencionadas por: B. ELIACHEVITCH, La personnalité juridique en droit privé romain, Paris 1942, p. 141 ntt. 151-152; M. SAlAZAR ReVuelta, La gratuidad del "mutuum" en el derecho romano, Jaén 1999, p. 335 s.; G.L. GREGORI, Nomina transcripticia e praedia subsignata: debiti, ipoteche e finanze locali a Trebvla Suffenatiom, en Il capitolo delle entrate nelle finanze municipali in Occidente ed in Oriente. Actes de la Xe Rencontre franco-italienne sur l'épigraphie du monde romain, Rome, 27-29 mai 1996, Rome 1999, p. 33 s.; J.F. Rodríguez NeILA, "Tabulae publicae". Archivos municipales y documentación financiera en las ciudades de la Bética, Madrid 2005, p. 125 ss. y notas. La causa mutui no se ve claramente en las dos inscripciones comentadas por GREGORI (vid. op. cit., p. 32) en las que se habla de créditos de la ciudad de Trebula Suffenatium. El caso de la institución alimentaria de Veleia presenta unas particularidades: el dinero entregado en mutuo probablemente no es municipal, sino fiscal, y el mutuario debe pagar al municipio de Veleia solamente las usurae; por otro lado el municipio está obligado a destinar las mismas al sustento de los pueri y de las puellae por medio del quaestor alimentorum; en este sentido vid. G. MAININO, La tabula alimentaria di Veleia fra politica, diritto ed evergetismo. Problemi e prospettive, en Archivio storico per le Province Parmensi 44 (1992), p. 13 ss., 21 (separ.); además, CH. CORBO, Paupertas. La legislazione tardoantica, Napoli 2006, p. 49 ss. 
cuales desde este punto de vista entraban en concurrencia con los argentarii; por lo tanto, los préstamos que aquí nos interesan son aquellos en los que el municipio, a través de sus representantes, asumía el papel de prestamista, acreedor. En cambio pasaremos por alto los casos que se refieren a la financiación ab externo de los municipia, o sea cuando los mismos tenían el papel de mutuario, deudor.

Por otro lado, es oportuno recordar que el mutuo público no tenía como función pública exclusivamente la búsqueda de la rentabilidad del dinero público; también se tomaban en consideración otros intereses públicos, como por ejemplo el decoro de la ciudad, o bien la necesidad de vivienda, cuando se financiaba, mediante mutuos públicos con usurae, la reconstrucción de un edificio privado ${ }^{3}$.

3 Cfr. D.39.2.46 (Paul. 1 sent.) = PS.1.1a.29-30: «Ad curatoris rei publicae officium spectat, ut dirutae domus a dominis extruantur. 1. Domum sumptu publico extructam, si dominus ad tempus pecuniam impensam cum usuris restituere noluerit, iure eam res publica distrahit». Sobre este texto cfr. G. CAMODECA, Ricerche sui curatores rei publicae, en ANRW II.13, Berlin - New York 1980, p. 466 s.; V. SCARANO USSANI, Le forme del privilegio. Beneficia e privilegia tra Cesare e gli Antonini, Napoli 1992, p. 158 s.; para J.M. RAINER, Bau- und nachbarrechtliche Bestimmungen im klassischen römischen Recht, Graz 1987, p. 297, en la época clásica se habría hecho referencia a un magistrado ciudadano, pero no al curator rei publicae; contra véase G. CAMODECA, I curatores rei publicae in Italia: note di aggiornamento, en Le quotidien municipal dans l'Occident romain. Actes du Colloque tenu à Clermont-Ferrand, du 19 au 21 octobre 2007 (dir. Cl. Berrendonner - M. 
Hay sin duda unas notas especificas respecto al mutuo entre particulares: por ejemplo, es posible pactar usurae también en nudo pacto [D.22.1.30 (Paul. lib. sing. reg.): «Etiam ex nudo pacto debentur civitatibus usurae creditarum ab eis pecuniarum»] ${ }^{4}, \mathrm{y}$ en general tenemos que preguntarnos si ese tipo de contrato público puede encuadrarse dentro del correspondiente tipo privatístico o bien puede tratarse de algo diferente. A este respecto quiero recordar que Vincenzo Arangio-Ruiz, en su destacado manual, prefería hablar de concesiones administrativas, incluyendo la tutela jurisdiccional de las mismas en el seno de la cognitio extra ordinem 5.

Podemos leer las fuentes relevantes, formulando las tres preguntas siguientes:

1) ¿Quién toma la decisión de invertir dinero público en préstamos?

2) ¿Quién elige el mutuario y según cuáles criterios?

Cébeillac-Gervasoni - L. Lamoine), Clermont Ferrand 2008, p. 515 y nt. 38. Por último, R. RODRíGUEz LóPEZ, Tres constituciones de Diocleciano sobre el urbanismo de finales del s. III d.C., en Estudios sobre Diocleciano (coord. A. Díaz Bautista), Madrid 2010, p. 256.

4 Cfr. V. De Villa, Le "usurae ex pacto" nel diritto romano, Roma 1937, p. 87 s.; G. GILIBERTI, Legatum kalendarii. Mutuo feneratizio e struttura contabile del patrimonio nell'età del principato, Napoli 1984, p. 12 e nt. 35; M. TALAMANCA, Istituzioni di diritto romano, Milano 1990, p. 545.

5 Cfr. V. ArangIo RuIZ, Istituzioni di diritto romano, Napoli $1998^{14}$ (rist.), p. $306 \mathrm{~s}$. 
3) ¿Quién controla desde un punto de vista administrativo y judicial la regularidad de la actuación pública? Sobre este último punto tenemos en realidad la mayoría de las incertidumbres ${ }^{6}$.

\section{Análisis de las fuentes relevantes.}

a) El rescripto de Vardacate.

El rescripto de Vardacate (I siglo d.C.) 7, municipio que se encontraba en la regio IX de Augusto (Italia del Norte-Oeste), en

6 Nuestras observaciones llegan hasta el fin de la época de los Severos. Según la mayoría de los autores sólo a partir de la segunda mitad del siglo III d.C. el curator rei publicae (funcionario implicado en el control de los mutuos municipales) se convierte de mandatario del emperador en magistrado ciudadano: cfr. F. JACQUES - J. SCHEID, Roma e il suo Impero. Istituzioni, economia, religione, Roma-Bari 19992, p. 344; W. ECK, L'Italia nell'Impero romano. Stato e amministrazione in epoca imperiale, Bari 1999, p. 228; adde F. CAMIA, I curatores rei publicae nella provincia d'Acaia, en MEFRA 119.2 (2007), p. 409; contra CL. LEPELLEY, Témoignages épigraphiques sur le contrôle des finances municipales par les gouverneurs à partir du règne de Dioclétien, en Il capitolo dell'entrate nelle finanze muncipali in Occidente ed in Oriente. Actes de la $X^{e}$ Rencontre franco-italienne sur l'épigraphie du monde romain - Rome, 27-29 mai 1996, Rome 1999, p. 242.

$7 \quad \mathrm{Al}$ respecto véase recientemente M. DE SIMONE, "Vas appellatus qui pro altero vadimonium promittebat". Per una lettura di Varro, De ling. lat., 6.74, en AUPA. 53 (2009), p. 195 s. (allí otras referencias bibliográficas). 
las líneas 7-9 («Pecuniae quae sine decurionum decreto erogatae sunt ab IIviris restitui rei publicae oportet qui eas dederunt sua sponte ita ut petitio eis sit adversus eos quibus crediderunt»; ed. L'Année épigraphique 44, 1947, n. 44, p. 21) nos informa de modo indirecto de la praxis legítima, esto es, que la entrega en mutuo de pecunia publica municipal tenía que estar basada en un decreto de autorización de los decuriones (los senadores locales). Si faltaba esa autorización, los magistrados que habían entregado el dinero público, se convertían en responsables con respecto a la colectividad municipal (res publica).

b) Cap. 79, Tab. Irnitana.

En el capítulo 79 de la lex Irnitana - «...item de pec[u]nia, quae communis municipum erit,... alienanda diminuenda eroganda mutua danda municipum nomine, deve remissione facienda ei quem municipibus eius municipi quid dare facere praestare oportebi[t], ne referto ad decuriones conscriptosve [c]um pauciores quam q[ui] tres quartas partes totius / numeri decurionum conscriptorumve explere possint aderunt...» - que no está lejana cronológicamente del rescripto de Vardacate, se confirma que es necesaria la autorización del senado local. Y además se prevé la intervención de una mayoría cualificada de los senadores (al menos los tres cuartos de la totalidad de los miembros de la 
asamblea) cuando se trata de decidir sobre la pecunia communis mutua danda municipum nomine 8.

c) Plin., Epist. X, 54-55.

Por lo que se refiere a la parte oriental del imperio, estamos así mismo informados de que era habitual el uso de préstamos municipales con inversión de dinero público. Es oportuno examinar para ello el siguiente fragmento:

Plin., Epist. X, 54-55: «C. PLINIUS TRAIANO IMPERATORI. 1 Pecuniae publicae, domine, providentia tua et ministerio nostro et iam exactae sunt et exiguntur; quae vereor ne otiosae iaceant. Nam et praediorum comparandorum aut nulla aut rarissima occasio est, nec inveniuntur qui velint debere rei publicae, praesertim duodenis assibus, quanti a privatis mutuantur. 2 Dispice ergo, domine, numquid minuendam usuram ac per hoc idoneos debitores invitandos putes, et, si nec sic reperiuntur, distribuendam inter decuriones pecuniam, ita ut recte rei publicae caveant; quod quamquam invitis et recusantibus minus acerbum erit leviore usura constituta.

8 Cfr. praecipue J.F. Rodríguez Neila, op. cit., p. 125 s.; 'pecunia communis' denota sin duda el dinero público municipal: en este sentido M. Talamanca, 'Ibi' in 'Lex Irnitana', 69, lin. 12-13: un contributo allo studio dell' 'agere de pecunia communi', en BIDR. 101-102 (1998-1999) [2005], p. 708; con respecto a la mayoría cualificada exigida vid. V. Marotta, Conflitti politici e governo provinciale, en Politica e partecipazione nelle città dell'impero (ed. F. Amarelli), Roma 2005, p. 165 nt. 149. 
TRAIANUS PLINIO. Et ipse non aliud remedium dispicio, mi Secunde carissime, quam ut quantitas usurarum minuatur, quo facilius pecuniae publicae collocentur. Modum eius, ex copia eorum qui mutuabuntur, tu constitues. Invitos ad accipiendum compellere, quod fortassis ipsis otiosum futurum sit, non est ex iustitia nostrorum temporum» 9.

En la epístola de Plinio el Joven, legatus Augusti pro praetore en la provincia de Ponto y Bitinia, dirigida al emperador Trajano el mismo gobernador le solicita autorización para bajar los intereses usurarios respecto al interés legal del 12\% atendiendo a la presumible dificultad de encontrar mutuarios en las ciudades de su provincia; y como alternativa le plantea la propuesta de obligar a los decuriones a recibir en préstamo aún con intereses rebajados. Según la opinión de Chantal Gabrielli 10 que comparto, no se trata aquí de dinero fiscal, sino de dinero municipal11, y merece la pena subrayar que en la

9 Sobre este texto cfr. recientemente $\mathrm{CH}$. CORBO, op. cit., p. 54 s.

10 Cfr. CH. Gabrielli, Pecuniae publicae...ne otiosae iaceant (Plin. epist. 10.54). Strategie finanziarie nell'amministrazione provinciale, en Gli Statuti Municipali (a cura di L. Capogrossi Colognesi - E. Gabba), Pavia 2006, p. 384 ss.

11 En cambio E. GABBA, Progetti di riforme economiche e fiscali in uno storico dell'età dei Severi, en Studi in onore di A. Fanfani I. Antichità e Alto Medioevo, Milano 1962, p. 63 s., tenía dudas sobre la naturaleza, municipal o bien fiscal, de la pecunia publica mencionada en la correspondencia de 
contestación del emperador la autonomía de las ciudades parece bastante limitada por el gobernador: en efecto, el mismo puede directamente establecer - dentro del límite máximo legal y en base al número de solicitudes de financiación - la tasa de interés que se aplicará entregando en mutuo el dinero municipal 12. Podemos preguntarnos si esta solución refleja unas particularidades de la provincia de Ponto-Bitinia 13, o bien si este poder del gobernador en el marco de las elecciones que se referían a las finanzas locales se ejercitaba también en otras provincias, limitando aquella autonomía de los senados locales

Plinio que estamos analizando. Véase también E. LO CASCIO, Il princeps e il suo impero. Studi di storia amministrativa e finanziaria romana, Bari 2000, p. $249 \mathrm{~s}$.

12 Es oportuno recordar que ya en la época tardo-republicana un título del edictum provinciale concernía las rationes civitatum: vid., entre otros, $\mathrm{R}$. CARDILLI, La «buona fede» come principio di diritto dei contratti. Diritto romano e America Latina, en Il ruolo della buona fede oggettiva nell'esperienza giuridica storica e contemporanea (Atti del Convegno internaz. di studi in onore di A. Burdese, a cura di L. Garofalo) I, Padova 2003, p. 290. Por lo que se refiere a las características del control sobre las finanzas municipales ejercido por el gobernador provincial, a la luz de las correspondencia entre Plinio y Trajano, cfr. C. BRÉLAZ, Motifs et circonstances de l'ingérence des autorités romaines dans les cités grecques sous le Principat, en Amministrare un impero. Roma e le sue province (a cura di A. Baroni), Trento 2007, p. 118 s.

13 En este sentido CH. Gabrielli, op. cit., p. 392 s.; vid. además V. MAROTTA, Ulpiano e l'impero II. Studi sui libri de officio proconsulis e la loro fortuna tardoantica, Napoli 2004, p. 180 e nt. 92. 
que hemos visto reconocida en la Tabula Vardacatensis y en la lex Irnitana. En cualquier caso, insisto, a la luz de la correspondencia entre Plinio y Trajano, resalta sin duda el uso habitual del mutuo para inversión («Pecuniae publicae...ne otiose iaceant») 14 .

d) Fragmentos de la jurisprudencia clásica.

En base a las fuentes jurídicas que atañen la época de los Severos, cabe decir que también durante esa misma época las ciudades invierten usualmente sus propios dineros en préstamos con intereses. De la compleja cuestión ventilada por Paulo - D. 22.1.11.pr. (Paul. 25 quaest.): «Gaius Seius qui rem publicam gerebat faeneravit pecuniam publicam sub usuris solitis...» 15 - conocemos que un administrador público (magistrado o curator), llamado Gaius Seius, había entregado en mutuo dinero municipal "sub usuris solitis". Tal vez se le había prometido el pago de las usurae mediante una stipulatio 16, pero también el nudo pacto hubiera sido válido según las palabras del mismo

14 Otra frecuente forma de inversión es la compra de fincas y sucesivo arrendamiento: cfr. CH. GABRIELLI, op. cit., p. 391.

15 Cfr. a propósito del texto M. TALAMANCA, Particolarismo normativo ed unità della cultura giuridica nell'esperienza romana, en Diritto generale e diritti particolari nell'esperienza storica. Atti del Congresso della Società italiana di storia del diritto (Torino, 19-21 novembre 1998), Roma 2001, p. 171 s.

16 Así A. BRICCHI, op. cit., p. 378. 
jurista (que se encuentran en el liber singularis regularum) 17, así que podemos vislumbrar desde este punto de vista una clara diferencia entre mutuo público y mutuo privado 18.

En los libros de officiis de Ulpiano, que consideramos en cierta medida obras de derecho administrativo (en base a lo que hoy en día incluye esta rama del ordenamiento jurídico) ${ }^{19}$ no

17 Cfr. supra, §1 y nt. 4.

18 El principio contrario en el ámbito de las relaciones entre particulares es expresado por el mismo Paulo (D.2.14.17.pr.: «Si tibi decem dem et paciscar, ut viginti mihi debeantur, non nascitur obligatio ultra decem: re enim non potest obligatio contrahi, nisi quatenus datum sit») y se reitera en P.S.2.14.1 («Si pactum nudum de praestandis usuris interpositum sit, nullius est momenti; ex nudo enim pacto inter cives Romanos actio non nascitur»). En doctrina véase G. CERVENCA, voz 'Usura' (dir. rom.), en Enc. Dir. 45 (1992), p. 1129; A. PetrucCI, Prime riflessioni su banca ed interessi nell'esperienza romana, en L'usura ieri ed oggi. Atti del Convegno, Foggia, 7-8 aprile 1995 (a cura di S. Tafaro), Bari 1997, p. 63; además P. Groeschler, Il 'mutuum cum stipulatione' e il problema degli interessi, en Quaderni Lupiensi di Storia e Diritto (a cura di F. Lamberti), Lecce 2009, p. 118. Por otro lado el mutuo de las civitates no tenía un régimen jurídico distinto respecto al mutuo entre particulares: a propósito de la extensión del senadoconsulto Macedoniano cfr. D.14.6.15 (Marc. 14 inst.) y el comentario de este texto de S. LONGO, Senatusconsultum Macedonianum. Interpretazione e applicazione da Vespasiano a Giustiniano, Torino 2012, p. 150 s.; adde (recensión del libro de S. Longo) F. LuCREZI, Ancora sul «Senatusconsultum Macedonianum», en RDR. 14 (2014), p. 9.

19 Cfr. A. Trisciuoglio, Percorsi semantici antichi e dogmatica giuspubblicistica moderna. Da 'officium' a 'ufficio', en Linguaggio e sistematica 
faltan menciones de mutuos municipales donde la parte pública se coloca en la posición de prestamista. En la obra dedicada al officium del curator rei publicae se requiere que el gobernador realice un cuidadoso control sobre el pago de las usurae en favor de las ciudades que se encuentran bajo su cognitio; e impresiona mucho la sugerencia hecha por el jurista sobre la manera de actuar con respecto a la recaudación: con relación a los debitores civitatis hay que comportarse de una manera inflexible pero también humana, comprensiva. En el mismo texto se atribuye todavía al gobernador provincial un control (no pienso que sea de carácter previo, sino sucesivo y de carácter judicial), en atención a las garantías proporcionadas por los mutuarios, probablemente con el fin de comprobar si los magistrados, o los curatores especiales, de la ciudad fuesen responsables de haber aceptado bienes, dados en prenda o hipoteca, inadecuados D.22.1.33 (Ulp. l. sing. de off. cur. rei publ.): «Si bene collocatae sunt pecuniae publicae, in sortem inquietari debitores non debent et maxime si parient usuras: si non parient, prospiciere rei publicae securitati debet praeses provinciae, dummodo non acerbum se exactorem nec contumeliosum praebeat, sed moderatum et cum efficacia benignum et cum instantia humanum: nam inter insolentiam incuriosam et diligentiam non ambitiosam multum interest. Praeterea

nella prospettiva di un romanista. Atti della Giornata di Studi in onore del Professor Lelio Lantella, Torino, 22 marzo 2013 (a cura di S. Masuelli e L. Zandrino), Napoli 2014, p. 39 y nt. 46. 
prospicere debet ne pecuniae publicae credantur sine pignoribus idoneis vel hypothecis» - 20.

En la misma obra de Ulpiano se reconoce, en base a constituciones imperiales21, aunque no están precisamente mencionadas, el poder probablemente del curator rei publicae 22 de promover la anulación de los "ambitiosa decreta" deliberados por parte de los decuriones, cuando los mismos decreta vayan en perjuicio de la caja municipal; y entre los varios casos donde se plantean "ambitiosa decreta" podríamos incluir también, por ejemplo, el de la entrega de dinero público al mutuario que no

20 Sobre D.22.1.33 véase A. DELL'ORO, I libri de officio nella giurisprudenza romana, Milano 1960, p. 224; G. CAMODECA, Ricerche cit., p. 469 nt. 69; F. JACQUES, Le privilège de liberté. Politique impériale et autonomie municipale dans les cités de l'Occident romain (161-244), Paris-Rome 1984, p. 291, 301 ss.; W. ECK, L'Italia nell'Impero romano cit., p. 224 s., nt. 129; V. MAROTTA, Ulpiano e l'impero II cit., p. 180. La responsabilidad del magistrado ciudadano mutuante por la aceptación de garantías inadecuadas puede inferirse del caso examinado por Modestino, discípulo de Ulpiano, en D.50.1.36.1 (Mod. 1 resp.); cfr. D. MANTOVANI, Il iudicium pecuniae communis. Per l'interpretazione dei capitoli 67-71 della lex Irnitana, en Gli Statuti Municipali (a cura di L. Capogrossi Colognesi - E. Gabba), Pavia 2006, p. 280 s.

21 Cfr. C.10.47.2 (impp. Diocl. et Maxim. Cassio): «Ordinis ambitiosa decreta sacris constitutionibus reprobantur».

22 A propósito de los problemas que se plantean tratando de atribuir sus competencias al curator rei publicae a la luz de los fragmentos extraídos del liber singularis de officio curatoris rei publicae de Ulpiano, vid. infra, § 3 . 
sea conveniente, así como el de la exención injustificada del pago de los intereses - D.50.9.4.pr.-1 (Ulp. l. sing. de off. cur. rei publ.): «Ambitiosa decreta decurionum rescindi debent, sive aliquem debitorem dimiserint sive largiti sunt. Proinde, ut solent, sive decreverint de publico [alicui]...certam quantitatem praestari, nihil valebit huiusmodi decretum». - 23. Con respecto a medidas semejantes en un fragmento de Calístrato se habla de rescissio por utilidad pública 24 .

23 Sobre D.50.9.4 véase: A. D'ORS, Epigrafía jurídica de la España romana, Madrid 1953, p. 280; A. Dell'OrO, I libri cit., p. 225 s.; D. NÖRR, Imperium und polis in der hohen Prinzipatszeit, München 19692, p. 25; M. BRUTTI, voz 'Invalidità' (storia), en Enc. Dir. 22 (1972), p. 569 nt. 49; G.G. ARCHI, Teodosio II e la sua codificazione, Napoli 1976, p. 82 nt. 60; G. CAMODECA, Ricerche cit., p. 470; F. JACQUES, Le privilège cit., p. 291, 303 ss.; V. MAROTTA, Multa de iure sanxit. Aspetti della politica del diritto di Antonino Pio, Milano 1988, p. 149 ss.; ID., Conflitti politici e governo provinciale cit., p. 192 s.; F. LAMBERTI, «Tabulae Irnitanae». Municipalità e «ius Romanorum», Napoli 1993, p. 114; G. COPPOLA, Cultura e potere. Il lavoro intellettuale nel mondo romano, Milano 1994, p. 334 ss.; A. PARMA, Sulla presenza di decreta decurionum nella pars tertia, Negotia, dei Fontes Iuris Romani Antejustiniani, en Revisione ed integrazione dei Fontes Iuris Romani Anteiustiniani (FIRA). Studi preparatori I. Leges (a cura di G. Purpura), Torino 2012, p. 234 nt. 41; L. Di PINTO, Cura studiorum. Tra pensiero giuridico e legislazione imperiale, Napoli 2013, p. 145 nt. 139.

24 Cfr. D.50.9.5 (Call. 2 de cognit.): «Quod semel ordo decrevit, non oportere id rescindi divus Hadrianus Nicomedensibus rescripsit nisi ex causa: id est si ad publicam utilitatem respiciat rescissio prioris decreti»; vid. recientemente R. 
Asimismo, en la obra de officio proconsulis el jurista de Tiro, tratando de los requisitos necesarios para desempeñar un cargo público electivo de la ciudad, se detiene sobre una específica categoría de sujetos, la de los debitores civitatis, aclarando quién está incluido en ella a la luz de un rescripto de Marco Aurelio y de Lucio Vero dirigido a Aufidius Herennianus. De forma que los debitores civitatis a quienes no se permite aspirar a los honores son solamente aquellos que dejaron deudas en la gestión del patrimonio ciudadano, pero no los mutuarios de la civitas que no han cumplido todavía sus obligaciones, ya que con respecto a ellos se considera que la ciudad queda adecuadamente garantizada por las prendas y las fianzas recibidas - D.50.4.6.1 (Ulp. 4 de off. proc.): «Debitores rerum publicarum ad honores invitari non posse certum est, nisi prius in id quod debetur rei publicae satisfecerint. Sed eos demum debitores rerum publicarum accipere debemus, qui ex administratione rei publicae reliquantur: ceterum si non ex administratione sint debitores, sed mutuam pecuniam a re publica acceperint, non sunt in ea causa, ut honoribus arceantur. Plane vice solutionis sufficit, ut quis aut pignoribus aut fideiussoribus idoneis caveat: et ita divi fratres Aufidio Herenniano rescripserunt» 25 -. Claro que este texto supone el uso del mutuo

SCEVOLA, 'Utilitas publica' II. Elaborazione della giurisprudenza severiana, Padova 2012, p. 379 s.

25 Cfr. D. MANTOVANI, Il iudicium pecuniae communis cit., p. 302 nt. 152; P. LEPORE, «Rei publicae polliceri». Un'indagine giuridico-epigrafica, Milano $2012^{2}$, p. 281 nt. 6 (allí otras referencias bibliográficas). 
municipal en la frase "sed mutuam pecuniam a re publica acceperint".

La cancillería imperial de la época de los divi fratres se revela muy activa, cuando se trata de reglamentar, a través de rescriptos, situaciones que, a mi juicio, pueden referirse también a mutuos de las civitates. En la colección de las constituciones imperiales de Papirio Justo, se halla un rescripto donde se relaciona la responsabilidad del curator ciudadano con el control que él tenía que llevar en torno a la buena salud del crédito de la civitas (por ejemplo, cuando acepta las garantías del mutuario y posteriormente no vigila la adecuación de las mismas). Y se dice que él es responsable por los avatares que se refieren al crédito público acontecidos en el ejercicio de su cargo, pero no por los ocurridos anteriormente, de los que, según parece, es responsable el curator anterior - D.50.8.12.6 (9) (Pap. Iust. 2 de const.): «Item (scil. impp. Antoninus et Verus) rescripserunt nominum, quae deteriora facta sunt tempore curatoris, periculum ad ipsum pertinere: quia vero antequam curator fieret, idonea non erant, aequum videri periculum ad eum non pertinere» 26

26 Aunque se habla propiamente de nomina sin aclarar la causa del crédito, creo que el rescripto pueda referirse también al mutuo. A propósito del segundo libro del de constitutionibus de Papirio Justo véase F. ARCARIA, Oratio Marci. Giurisdizione e processo nella normazione di Marco Aurelio, Torino 2003, p. 83. G. GILIBERTI, Legatum kalendarii cit., p. 12 nt. 34, opina que el curator mencionado es un curator kalendarii cargado de un munus. 
-. Y conocemos otras disposiciones imperiales sucesivas C.11.39.1 - imp. Alex. Severus 27; C.11.36.4 - impp. Dioclet. y Maxim.28 - las cuales quieren limitar la responsabilidad de los magistrados y de los curatores en caso de que los mutuarios de dinero público no cumplan con sus deberes.

\section{Observaciones finales.}

No hay duda, por lo tanto, que el mutuo público es un negocio muy empleado por los municipia entre el I y el III siglo d.C.; en cambio hay muchas incertidumbres acerca de las relaciones entre los diferentes poderes públicos implicados, y

27 C.11.39.1 (imp. Alex. A. Octavio): «Fenoris rei publicae, quod non tua culpa perditum esse apparuerit, sufficit sortis damnum, non etiam usurarum sustinere»; cfr. K.F.F. KNIEP, Die Mora des Schuldners nach Römischem und heutigem Recht I, Rostock 1871, p. 256.

28 C.11.36.4 (impp. Dioclet. et Maxim. AA. Dioni): «Cum te et collegam tuum magistratus vestri tempore publicum fenus collocasse proponas nec potuisse debitum a quibusdam rei publicae restitui, cum paratus sis pro te id quod solus gessisti satis rei publicae facere, consequens est rem publicam, si separati actus vestri fuerint, contra successores collegae tui vel rerum eius possessores prius ob personam eius progredi et, si solida indemnitas rei publicae servata non fuerit, nominatores eius interpellare ac postremo loco, si quid ex nomine collegae tui defuerit, ob culpae nexum te interpellare. Priores nominatores enim veluti fideiussores placuit obstringi»; cfr. D. MANTOVANI, Il iudicium pecuniae communis cit., p. 281 nt. 77. 
por consiguiente acerca del nivel de autonomía reconocido en este ámbito a las civitates. En general podríamos decir que la elección de invertir, a través de un mutuo, el dinero público municipal correspondía al senado local, y los magistrados ciudadanos debían concertar el contrato con los mutuarios que habían entregado adecuadas garantías. Pero ¿cabe creer que los senados locales tenían una absoluta libertad en esa elección? O bien ¿los mismos estaban condicionados por los curatores rei publicae y los gobernadores provinciales? 29.

Me limito, para terminar, a indicar todavía unos datos que debemos tener en cuenta para solucionar estos problemas. 1) No en todas las ciudades estaban presentes los comisarios imperiales (i.e. los curatores rei publicae) encargados de revisar las cuentas municipales ${ }^{30}$. 2) Tenemos dentro del Digesto de

29 Estos últimos, por otro lado, eligen los curatores kalendarii - cfr. D.50.8.12.4 (rescripto de Marco Aurelio y Lucio Vero); G. GILIBERTI, Legatum kalendarii cit., p. 11; B. SIRKS, The management of public loans of towns (the cura kalendarii) and of their finances in general, en AARC. XII Conv. internaz. in onore di M. Sargenti, Napoli 1998, p. 380; W. ECK, L'Italia nell'Impero romano cit., p. 229 s.; V. MAROTTA, Ulpiano e l'impero II cit., p. 180 nt. 94 -que están colocados tal vez en una posición subalterna respecto a los curatores rei publicae; en este sentido vid. L. JAPELLA CONTARDI, Un esempio di 'burocrazia municipale': i curatores kalendarii, en Epigraphica 39 (1977), p. 84 s.

30 Cfr. F. JACQUES - J. SCHEID, Roma e il suo Impero cit., p. 341 s. Sobre el curator rei publicae en la época que hemos considerado vid., entre muchos otros, F. De MARTINo, Storia della costituzione romana IV.2, Napoli 1975², p. 
Justiniano muy pocos fragmentos de la obra de Ulpiano dedicada a las funciones del curator rei publicae; y además en ellos nunca está mencionado este funcionario; en cambio se hace referencia al gobernador provincial ${ }^{31}$. La fuente que habría sido la más importante para conocer la función del curator rei publicae (a la luz de las constituciones imperiales) con respecto también a los mutuos municipales, desafortunadamente no dice nada. Así que no podemos decir si el curator rei publicae tenía que emitir una opinión previa al senado, antes de la decisión de entregar dinero público en mutuo, o bien si el mismo funcionario podía solamente promover la anulación de la decisión ex post, por si acaso la misma fuese un "ambitiosum decretum" y en este caso si su intervención ocurría en el ámbito de un juicio que se celebraba ante el gobernador provincial ${ }^{32}$.

694 s.; F. CAMACHo, "Cvrator rei publicae», en Estudios en homenaje al Prof. Juan Iglesias III, Madrid 1988, p. 1159 ss.; M. SARTORI, Osservazioni sul ruolo del curator rei publicae, en Athenaeum 67 (1989), p. 5 ss.; G.P. BURTON, The Roman Imperial State, Provincial Governors and the Public Finances of Provincial Cities. 27 B.C. - A.D. 235, en Historia 53 (2004), p. 336 ss.; con especial atención a la provincia de Acaya, F. CAMIA, I curatores rei publicae cit., p. 409 ss.

31 Sobre las posibles razones vid. G. CAMODECA, Ricerche cit., p. 456 ss.; F. JACQUES, Le privilège cit., p. 291, 302; W. ECK, L'Italia nell'Impero romano cit., p. 209 s., 224; E. LO CASCIO, Il princeps e il suo impero cit., p. 28.

32 De momento me parece la solución más probable; contra G. MANCINI, voz 'curator reipublicae o civitatis', en DE RUGGIERO, DE. II.2 (Roma 1910), p. 
Por otro lado, desde un punto de vista metodológico un discurso excesivamente general sobre este tema en el período que hemos considerado podría no estar conforme a la realidad histórica, ya que cada ciudad podía ser administrada de una manera diferente y con más o meno autonomía respecto a los poderes centrales, según sus particularidades jurídicas, sociales y económicas ${ }^{33}$.

\section{Bibliografía}

V. ARANGIO RUIZ, Istituzioni di diritto romano, Napoli $19988^{14}$.

1365, que, en atención a D.50.9.4.1, piensa en una anulación directa por parte del curator rei publicae; en el mismo sentido G.A. CECCONI, Crisi e trasformazioni del governo municipale in Occidente fra IV e VI secolo, en Die Stadt in der Spätantike - Niedergang oder Wandel? Akten des intern. Kolloquiums in München am 30. und 31. Mai 2003, hrsg. von J.-U. Krause - Chr. Witschel, Stuttgart 2006, p. 293 nt. 25. Podríamos estar en verdad en el marco de aquellas "apelaciones reformadoras" que no concernían a sentencias judiciales y han sido investigadas recientemente por V. MAROTTA, Conflitti politici e governo provinciale cit., p. 157 ss., vid. en particular p. 193.

33 Cfr., por ejemplo, el régimen especial que tenía la colonia de Apamea en Bitinia: Plin., Epist. 10.47-48; L. CRACCO RuGGINI, La città imperiale, en Storia di Roma IV. Caratteri e morfologie (direz. A. Schiavone), Torino 1989, p. 236 s.; E. LO CASCIO, La dimensione finanziaria, en Gli Statuti Municipali (a cura di L. Capogrossi Colognesi - E. Gabba), Pavia 2006, p. 685; G.P. BURTON, The Roman Imperial State cit., p. 318. 
F. ARCARIA, Oratio Marci. Giurisdizione e processo nella normazione di Marco Aurelio, Torino 2003.

G.G. ARCHI, Teodosio II e la sua codificazione, Napoli 1976.

C. BRÉLAZ, Motifs et circonstances de l'ingérence des autorités romaines dans les cités grecques sous le Principat, en Amministrare un impero. Roma e le sue province (a cura di A. Baroni), Trento 2007.

A. BRICCHI, Amministratori ed actores. La responsabilità nei confronti dei terzi per l'attività negoziale degli agenti municipali, en Gli Statuti Municipali (a cura di L. Capogrossi Colognesi - E. Gabba), Pavia 2006.

G.P. BuRTON, The Roman Imperial State, Provincial Governors and the Public Finances of Provincial Cities. 27 B.C. - A.D. 235, en Historia 53 (2004).

F. CAMACHO, "Curator rei publicae», en Estudios en homenaje al Prof. Juan Iglesias III, Madrid 1988. 
F. CAMIA, I curatores rei publicae nella provincia d'Acaia, en MEFRA. 119.2 (2007).

G. CAMODECA, Ricerche sui curatores rei publicae, en ANRW. II.13, Berlin - New York 1980.

G. CAMODECA, I curatores rei publicae in Italia: note di aggiornamento, en Le quotidien municipal dans l'Occident romain. Actes du Colloque tenu à Clermont-Ferrand, du 19 au 21 octobre 2007 (dir. Cl. Berrendonner - M. Cébeillac-Gervasoni L. Lamoine), Clermont Ferrand 2008.

R. CARDILl, La «buona fede» come principio di diritto dei contratti. Diritto romano e America Latina, en Il ruolo della buona fede oggettiva nell'esperienza giuridica storica e contemporanea (Atti del Convegno internaz. di studi in onore di A. Burdese, a cura di L. Garofalo) I, Padova 2003.

G.A. CECCONI, Crisi e trasformazioni del governo municipale in Occidente fra IV e VI secolo, en Die Stadt in der Spätantike Niedergang oder Wandel ? Akten des intern. Kolloquiums in München am 30. und 31. Mai 2003, hrsg. von J.-U. Krause Chr. Witschel, Stuttgart 2006. 
G. CervencA, voz 'Usura' (dir. rom.), en Enc. Dir. 45 (1992).

G. CoppolA, Cultura e potere. Il lavoro intellettuale nel mondo romano, Milano 1994.

CH. Corbo, Paupertas. La legislazione tardoantica, Napoli 2006.

L. Cracco Ruggini, La città imperiale, en Storia di Roma IV. Caratteri e morfologie (direz. A. Schiavone), Torino 1989.

A. Dell'Oro, I libri de officio nella giurisprudenza romana, Milano 1960.

M. DE SIMONE, "Vas appellatus qui pro altero vadimonium promittebat". Per una lettura di Varro, De ling. lat., 6.74, en AUPA. 53 (2009).

A. D'ORS, Epigrafía jurídica de la España romana, Madrid 1953.

F. De Martino, Storia della costituzione romana IV.2, Napoli $1975^{2}$.

V. DE VILLA, Le "usurae ex pacto" nel diritto romano, Roma 1937. 
L. DI PINTO, Cura studiorum. Tra pensiero giuridico e legislazione imperiale, Napoli 2013.

W. ECK, L'Italia nell'Impero romano. Stato e amministrazione in epoca imperiale, Bari 1999.

B. ELIACHEVITCH, La personnalité juridique en droit privé romain, Paris 1942.

E. GABBA, Progetti di riforme economiche e fiscali in uno storico dell'età dei Severi, en Studi in onore di A. Fanfani I. Antichità e Alto Medioevo, Milano 1962.

CH. Gabrielli, Pecuniae publicae...ne otiosae iaceant (Plin. epist. 10.54). Strategie finanziarie nell'amministrazione provinciale, en Gli Statuti Municipali (a cura di L. Capogrossi Colognesi - E. Gabba), Pavia 2006.

G. GILIBERTI, Legatum kalendarii. Mutuo feneratizio e struttura contabile del patrimonio nell'età del principato, Napoli 1984. 
G.L. GREGORI, Nomina transcripticia e praedia subsignata: debiti, ipoteche e finanze locali a Trebvla Suffenativm, en Il capitolo delle entrate nelle finanze municipali in Occidente ed in Oriente. Actes de la Xe Rencontre franco-italienne sur l'épigraphie du monde romain, Rome, 27-29 mai 1996, Rome 1999.

P. Groeschler, Il 'mutuum cum stipulatione' e il problema degli interessi, en Quaderni Lupiensi di Storia e Diritto (a cura di F. Lamberti), Lecce 2009.

F. JACQUES, Le privilège de liberté. Politique impériale et autonomie municipale dans les cités de l'Occident romain (161-244), ParisRome 1984.

F. JACQUES - J. SCHEID, Roma e il suo Impero. Istituzioni, economia, religione, Roma-Bari 19992.

L. JAPELla CONTARDI, Un esempio di 'burocrazia municipale': i curatores kalendarii, en Epigraphica 39 (1977).

K.F.F. KNIEP, Die Mora des Schuldners nach Römischem und heutigem Recht I, Rostock 1871. 
F. LAMBERTI, «Tabulae Irnitanae». Municipalità $e$ «ius Romanorum», Napoli 1993.

CL. LEPELLEY, Témoignages épigraphiques sur le contrôle des finances municipales par les gouverneurs à partir du règne de Dioclétien, en Il capitolo dell'entrate nelle finanze muncipali in Occidente ed in Oriente. Actes de la Xe Rencontre franco-italienne sur l'épigraphie du monde romain - Rome, 27-29 mai 1996, Rome 1999.

P. LEPORE, «Rei publicae polliceri». Un'indagine giuridico-epigrafica, Milano 2012².

E. Lo CASCIO, Il princeps e il suo impero. Studi di storia amministrativa e finanziaria romana, Bari 2000.

E. Lo CASCIO, La dimensione finanziaria, en Gli Statuti Municipali (a cura di L. Capogrossi Colognesi - E. Gabba), Pavia 2006.

S. LONGO, Senatusconsultum Macedonianum. Interpretazione e applicazione da Vespasiano a Giustiniano, Torino 2012.

F. LUCREZI, Ancora sul «Senatusconsultum Macedonianum», en RDR. 14 (2014). 
G. MAININO, La tabula alimentaria di Veleia fra politica, diritto ed evergetismo. Problemi e prospettive, en Archivio storico per le Province Parmensi 44 (1992).

G. MANCINI, voz 'curator reipublicae o civitatis', en DE RUGGIERO, DE. II.2 (Roma 1910).

D. Mantovani, Il iudicium pecuniae communis. Per l'interpretazione dei capitoli 67-71 della lex Irnitana, en Gli Statuti Municipali (a cura di L. Capogrossi Colognesi - E. Gabba), Pavia 2006.

V. Marotta, Multa de iure sanxit. Aspetti della politica del diritto di Antonino Pio, Milano 1988.

V. MARotTA, Ulpiano e l'impero II. Studi sui libri de officio proconsulis e la loro fortuna tardoantica, Napoli 2004.

V. Marotta, Conflitti politici e governo provinciale, en Politica e partecipazione nelle città dell' impero (ed. F. Amarelli), Roma 2005. 
D. NÖRR, Imperium und polis in der hohen Prinzipatszeit, München 19692.

A. PARMA, Sulla presenza di decreta decurionum nella pars tertia, Negotia, dei Fontes Iuris Romani Antejustiniani, en Revisione ed integrazione dei Fontes Iuris Romani Anteiustiniani (FIRA). Studi preparatori I. Leges (a cura di G. Purpura), Torino 2012.

A. Petrucci, Prime riflessioni su banca ed interessi nell'esperienza romana, en L'usura ieri ed oggi. Atti del Convegno, Foggia, 7-8 aprile 1995 (a cura di S. Tafaro), Bari 1997.

J.M. RAINER, Bau- und nachbarrechtliche Bestimmungen im klassischen römischen Recht, Graz 1987.

R. RODRÍGUEZ LÓPEZ, Tres constituciones de Diocleciano sobre el urbanismo de finales del s. III d.C., en Estudios sobre Diocleciano (coord. A. Díaz Bautista), Madrid 2010.

J.F. Rodríguez NeILA, "Tabulae publicae". Archivos municipales y documentación financiera en las ciudades de la Bética, Madrid 2005.

M. SAlazAr Revuelta, La gratuidad del "mutuum" en el derecho romano, Jaén 1999. 
M. SARTORI, Osservazioni sul ruolo del curator rei publicae, en Athenaeum 67 (1989).

V. SCARANO USSANI, Le forme del privilegio. Beneficia e privilegia tra Cesare e gli Antonini, Napoli 1992.

R. SCEVOlA, 'Utilitas publica' II. Elaborazione della giurisprudenza severiana, Padova 2012.

B. SIRKS, The management of public loans of towns (the cura kalendarii) and of their finances in general, en AARC. XII Conv. internaz. in onore di M. Sargenti, Napoli 1998.

M. TALAMANCA, Istituzioni di diritto romano, Milano 1990.

M. TAlamancA, Particolarismo normativo ed unità della cultura giuridica nell'esperienza romana, en Diritto generale e diritti particolari nell'esperienza storica. Atti del Congresso della Società italiana di storia del diritto (Torino, 19-21 novembre 1998), Roma 2001. 
M. TalamancA, 'Ibi' in 'Lex Irnitana', 69, lin. 12-13: un contributo allo studio dell' 'agere de pecunia communi', en BIDR. 101-102 (1998-1999) [2005].

A.TORRENT, Financiación externa de los «municipia». Lex Irnitana cap. 80, en RDR. 10 (2010).

A. TRISCIUOGLIO, Societas publicanorum e aspetti della responsabilità esterna [Atti del Seminario di studi su: "Societas. Strumento di organizzazione pubblica e privata" (Sassari, 4-5 maggio 2012)], en Diritto@Storia 11 (2013).

A. TRIScIUOGLIO, Percorsi semantici antichi $e$ dogmatica giuspubblicistica moderna. Da 'officium' a 'ufficio', en Linguaggio e sistematica nella prospettiva di un romanista. Atti della Giornata di Studi in onore del Professor Lelio Lantella, Torino, 22 marzo 2013 (a cura di S. Masuelli e L. Zandrino), Napoli 2014.

M. TUCCI, L'amministrazione tra pubblico e privato e il principio di legalità dall'antichità ai giorni nostri. Aspetti ricostruttivi e prospettive di sviluppo, Milano 2008. 